ARTICLE

\title{
State-of-the-art augmented NLP transformer models for direct and single-step retrosynthesis
}

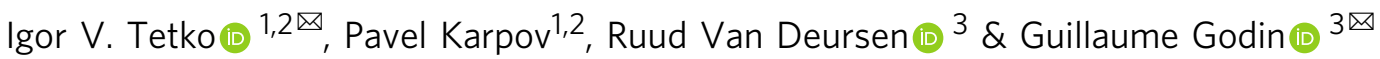

We investigated the effect of different training scenarios on predicting the (retro)synthesis of chemical compounds using text-like representation of chemical reactions (SMILES) and Natural Language Processing (NLP) neural network Transformer architecture. We showed that data augmentation, which is a powerful method used in image processing, eliminated the effect of data memorization by neural networks and improved their performance for prediction of new sequences. This effect was observed when augmentation was used simultaneously for input and the target data simultaneously. The top-5 accuracy was $84.8 \%$ for the prediction of the largest fragment (thus identifying principal transformation for classical retro-synthesis) for the USPTO-50k test dataset, and was achieved by a combination of SMILES augmentation and a beam search algorithm. The same approach provided significantly better results for the prediction of direct reactions from the single-step USPTO-MIT test set. Our model achieved $90.6 \%$ top- 1 and $96.1 \%$ top- 5 accuracy for its challenging mixed set and $97 \%$ top- 5 accuracy for the USPTO-MIT separated set. It also significantly improved results for USPTO-full set single-step retrosynthesis for both top-1 and top-10 accuracies. The appearance frequency of the most abundantly generated SMILES was well correlated with the prediction outcome and can be used as a measure of the quality of reaction prediction.

\footnotetext{
${ }^{1}$ Institute of Structural Biology, Helmholtz Zentrum München-Research Center for Environmental Health (GmbH), Ingolstädter Landstraße 1, D-85764 Neuherberg, Germany. ${ }^{2}$ BIGCHEM GmbH, Valerystr. 49, D-85716 Unterschleißheim, Germany. ${ }^{3}$ Firmenich International SA, D-Lab by Firmenich, Rue de la Bergère 7, CH-1242 Meyrin-Satigny, Switzerland. ${ }^{凶}$ email: i.tetko@helmholtz-muenchen.de; guillaume.godin@firmenich.com
} 
T o synthesize an organic compound is to solve a puzzle with many pieces and potentially several pieces missing. Here, the pieces are single reactions, and finding their sequential combination to create a final product is the retrosynthesis task.

The success of the logic of organic synthesis developed by Corey et al. ${ }^{1,2}$ triggered the development of computer programs aiming to find appropriate ways to synthesize a molecule. The first retrosynthesis program LHASA ${ }^{2}$ utilizes a template-based ${ }^{3,4}$ approach. Every template (rule, synthon) in a curated database of known transformations is sequentially applied to a target molecule, and then sets of reagents are selected according to a specified strategy. Reagents, in turn, undergo the same decompositions until a set of commercially available compounds is found. Retrosynthesis always has multiple routes-a retrosynthetic treeending with different starting materials. Thus, a practical algorithm for retrosynthesis has to solve not only the rule acquisition and selection problem but also has capabilities to effectively navigate this tree ${ }^{5}$, taking into account different strategies. These tasks relate directly to artificial intelligence strategies ${ }^{6-8}$.

Due to the difficulty of maintaining template databases, most projects dependent on them, including LHASA, did not become widely used tools. The only major exception is, perhaps, the program Synthia ${ }^{\mathrm{mo}}$ (previously CHEMATICA ${ }^{9}$ ) which is a successful commercial product. In the Synthia $a^{\text {tw }}$ program, rules are automatically extracted from atom-mapped reaction examples ${ }^{10}$. However, there is an ambiguity in the mapping definition and, more importantly, the automatic rule does not take into account other undefined possible reactive centers in a molecule. Applying such transformations may result in molecules that fail to react as predicted, e.g., "out-of-scopes" and special care to filter out these cases has to be taken ${ }^{5}$. An alternative approach for the extraction of these rules is to apply a data-driven deep learning technique that corresponds to a machine learning approach where an algorithm (usually in the form of a neural network) is trained on the raw data. After the training finishes, the network contains all the implicitly encoded features (rules) of the corresponding input via its parameters. Works on reaction prediction outcomes ${ }^{11}$ and retrosynthesis ${ }^{12,13}$ showed the feasibility of a symbolic approach, where reactions are written as SMILES ${ }^{14}$ strings as in a machine translation. The product is written in the "source language", whereas the set of reactants is written in the "target language". For the "reaction translation" task both languages, however, are SMILES strings, having the same alphabet and grammar. The first works on symbolic (retro)synthesis ${ }^{12,15}$ were carried out with Seq2Seq ${ }^{16}$ models following robust and more easy to train natural language processing (NLP) transformer approaches ${ }^{17,18}$ that bring state-of-the-art results ${ }^{11,19}$. Meanwhile other approaches based on similarity ${ }^{20}$, convolutional ${ }^{21-23}$, and graphs ${ }^{24,25}$ show promising results.

The SMILES representation of molecules is ambiguous. Though the canonicalization procedure exists ${ }^{26}$, it has been shown that models benefit from using a batch of random SMILES (augmentation) during training and inference ${ }^{27-30}$. Recently, such augmentation was also applied to reaction modeling $11,18,31,32$. The augmented (also sometimes called "random") SMILES are all valid structures with the exception that the starting atom and the direction of the graph enumerations are selected randomly.

In this article, we scrutinize the various augmentation regimes and show that augmentation leads to better performance compared to the standard beam search inference or evaluation of the model under different temperatures. We clearly mention that our study is to predict single-step and not multi-step retrosynthesis, which has been also targeted using transformer ${ }^{33,34}$. We show that by using more complicated data augmentation strategies we decrease overfitting ${ }^{35}$ of neural networks and increase their accuracy to achieve top performances for both direct and retro-synthesis. We observe that the harder are the data to train the model, the better it will predict new ones. Moreover, we introduce a new measure MaxFrag accuracy for the prediction of the largest fragment (thus identifying principal transformation for classical retro-synthesis).

\section{Results}

The baseline dataset contained only canonical SMILES. The other datasets also contained SMILES, augmented as described in the section Supplementary methods. Four different scenarios were used to augment training set sequences. Namely, we used augmentation of products only $(\mathrm{xN})$, augmentation of products and reactants/reagents $(\mathrm{xNF})$, augmentation of products and reactants/reagents followed by shuffling of the order of reactant/ reagents (xNS), and finally mixed forward/reverse reactions, where each retrosynthesis reaction from xNS was followed by the inverse (forward synthesis) reaction (xNM). Only the simplest augmentation $\mathrm{xN}$ was used for test sets because no information about reactant/reagents could be used for the retrosynthesis prediction. At least one copy of canonical SMILES for each reaction was present in all augmentation scenarios.

Reaction synthesis data. We used a training set filtered from USPTO databas $\mathrm{e}^{36}$ containing $50 \mathrm{k}$ reactions classified into 10 reaction types. We used splitting proposed by Liu et al. ${ }^{12}$ and divided it into 40,5 , and $5 \mathrm{k}$ reactions for the training, validation, and test sets, respectively. As in the previous study ${ }^{13}$, after observing that early stopping using the validation set did not improve model test accuracy (the model performance for each of the sets was monotonically increasing with number of iterations, see Supplementary Fig. 1), we combined the training and the validation sets into a combined training set. The $5 \mathrm{k}$ test reactions were predicted only once the model training was finished and were not used at any stage of the model development. In a similar way we joined training and validation sets of USPTO-MIT 22 dataset for direct reaction prediction. In order to provide a more straightforward comparison with results of the previous studies we also reported performances when developing models using only the respective training sets. Moreover a model with the largest published USPTO-full set ${ }^{24}$ was also developed.

Analysis of canonical datasets. The development of a model with canonical SMILES ( $\mathrm{x} 1$ ) as the training set provided $40.9 \%$ accuracy for prediction of the canonical test set. An attempt to use this model to predict the augmented test set $(\mathrm{x} 5, \mathrm{x} 10)$, resulted in much lower top- 1 predictions of $23.3 \%$ and $18.4 \%$, respectively. This result was to be expected, because the model trained with only canonical sequences was not able to generalize and predict augmented SMILES, which use different styles of molecular representation.

Augmentation of products only $(\mathrm{xN})$. The augmentation of the products (input data), with just one additional augmented SMILES $x 2$, increased top- 1 accuracy to $43.7 \%$ for the test data composed of canonical sequences. Increasing the number of augmentations in the training set did not increase the top-1 prediction accuracy. Thus, the augmentation of the training set with just one random SMILES contributed the best performance. This result is in concordance with another study where only one random SMILES was used to augment data ${ }^{18}$.

Analysis of character and exact sequence-based prediction accuracy. To better understand the model training, we also developed several models where approximately $10 \%$ of the dataset did not participate in training but was used to monitor its prediction performance. Different from the test set, which tested 


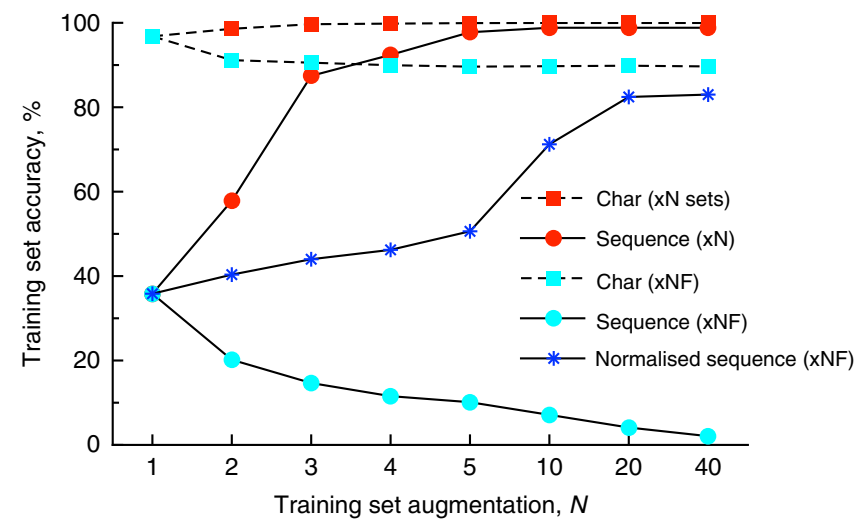

Fig. 1 Character and exact sequence-based accuracies calculated for the monitoring set. The transformer memorized the target sequences if the target sequences were all canonical SMILES (red dots). It also reasonably predicted the sequence composition for randomized target SMILES (cyan rectangle, dashed) but its performance decreased for prediction of exact full SMILES (cyan circle). The performance normalized by the percentage of canonical sequences increased with the number of augmentations, $N$, since some of the random sequences were canonical ones.

the performance of models when predicting a new reaction, the monitoring set tested the ability of the Transformer to predict different SMILES generated for the same reaction. The Transformer was able to recognize different representations of the same reaction. For example, when training $\mathrm{x} 1$, the character and exact sequence-based accuracies when predicting the monitoring sequences were $96.5 \%$ and $34.5 \%$, respectively. The final performance for the test set, $40.9 \%$, was higher because some reaction products from the transformer provided noncanonical SMILES, which were correctly matched after transformation to canonical ones. When using augmented training sequences (x10), the accuracies increased to $99.97 \%$ and $98.9 \%$, for character and exact sequence-based accuracy, respectively (see Fig. 1). The transformer recognized different representations of SMILES for reactants and reagents of the same training set reaction, and was able to exactly restore the target products which were memorized. Demonstrably, it was also able to memorize any random sequences. To show this, we used a random SMILES sequence (xNR set in Supplementary Tables 1 and 2, and Supplementary Fig. 2) instead of the canonical sequences as the target for prediction. While this task was more difficult and took more epochs to train, the transformer was able to perfectly memorize random sequences. Since the SMILES prediction target was random, the transformer was not able to learn canonicalization rules on how to write the target. Despite this fact, it still calculated a top-1 prediction accuracy of $26.8 \%$ for the test set which was, however, significantly lower compared to the $42.2 \%$ achieved using the $\mathrm{x} 10$ dataset with canonical sequences as the target.

Augmentation of reactants and reagents. A boost of the Transformer performance was observed when, in addition to products, i.e., the inputs SMILES, we also augmented the target SMILES, i.e., reactants and reagents. This task was more difficult for the transformer, which resulted in a drop in both character and sequence based scores for monitoring sequences during the training stage. For example, when using the training dataset with one augmented SMILES, x2F, the character based accuracy dropped to $91.3 \%$, which was lower than $98.6 \%$ calculated with the $\mathrm{x} 2$ dataset composed of canonical product SMILES (Fig. 1). For a larger number of augmentations, the character-based accuracy converged to a plateau, e.g., $89.96 \%$ and $89.67 \%$ for the

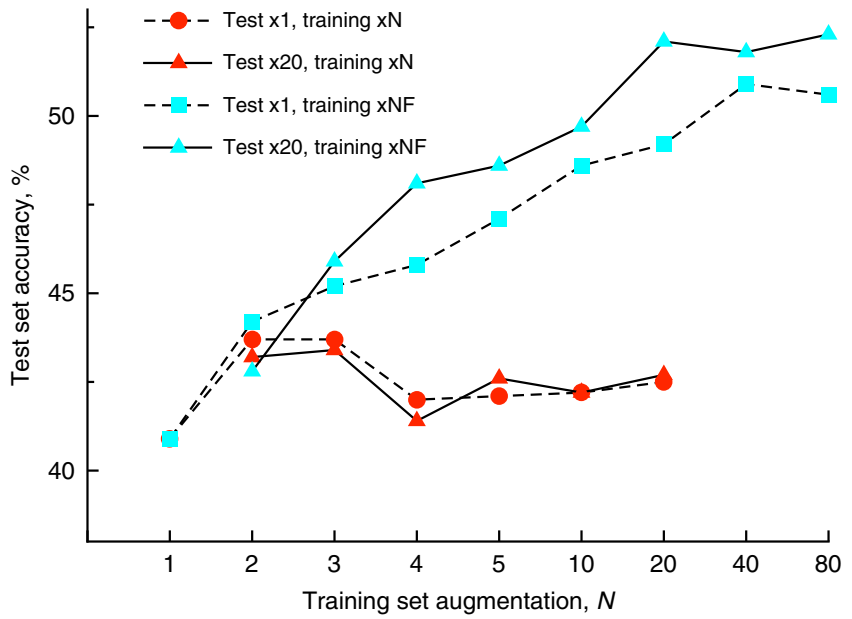

Fig. 2 Top-1 performance of models developed with different number of augmentation (shown on $x$-axis) and different augmentation scenarios applied to both test and training sets (red color: only products were augmented; cyan color: full reactions were augmented). The use of the large number of augmentations for the test set (solid lines) improved prediction accuracy for models developed with augmentation of full reactions but did not influence the performance of models where only input data were augmented.

$\mathrm{x} 5 \mathrm{~F}$ and $\mathrm{x} 20 \mathrm{~F}$ training sets, respectively. The character-based accuracy was calculated as the percentage of exact matches between target and predicted sequences, e.g., "CCCCN" and "NCCCC" have an accuracy of $80 \%$, despite being the same SMILES but written from different starting atoms. Thus despite the fact that the Transformer faced a prediction of random SMILES, it was still able to provide a reasonable prediction of their character composition.

However, of course, the transformer was not able to predict the exact random product SMILES. This resulted in a decrease in sequence-based accuracy based on the number of augmentations for xNF training datasets (Fig. 1, cyan circle). Still the transformer was able to predict some of the sequences, which corresponded to the subset of canonical sequences in the monitoring set. Interestingly, the sequence accuracy normalized to the percentage of canonical SMILES in the monitoring sets increased with the number of augmentations since some randomly generated sequences were canonical SMILES.

Top-1 performance analysis. For augmentations with 1 or 2 random SMILES, the top-1 prediction performance of the models trained with augmentation of reactants and reagents only, $\mathrm{xN}$, and full reaction augmentation, xNF, were similar. For a larger number of augmentations the models trained with xNF sets had systematically better performances than those developed with $\mathrm{xN}$ sets (Fig. 2). The training with the $\mathrm{x} 80 \mathrm{~F}$ set provided the highest top-1 performance of $52.3 \%$ when this model was applied to the test set generated with $\times 20$ number of augmented sequences. While it was possible that further increase in the augmentations could still increase the top-1 performance, we did not perform such calculations due to limitations on available computational resources.

Shuffling order of reactants. In addition to augmenting the full reaction, we also randomly shuffled the orders of reactants (see xNS set description in Supplementary Tables 1 and 2). The effect of this additional data perturbation improved top-1 performance to $53.1 \%$ for the $\times 20$ S training dataset applied to the test set with the same number of augmentations (Supplementary Fig. 3). 
Further increasing the number of augmentations resulted in the loss of top-1 prediction accuracy.

Shuffling and mixing of retrosynthesis and direct reactions. The training of retrosynthesis and direct reactions simultaneously could create a mixed representation of latent space and further increase the ability of the transformer to make generalizations. We tested this hypothesis by combining direct and reverse reactions in one training set by reversing the order of product/ reactant + reagents and adding a dot to distinguish direct reactions (see e.g., Supplementary Table 2, x2M augmentation). Contrary to previous analysis, which required 20 augmentations of training set sequences to achieve the highest performance, the mixed dataset achieved it with only 10 augmentations (Supplementary Fig. 3). Since the mixed dataset also included direct reactions, it had the same number of 19 augmented SMILES per canonical SMILES as in the previous analyses. Thus, this number of augmentations was optimal for the training of the transformer. A smaller number of augmentations did not allow it to fully explore the data diversity while a larger number created too much noise and made it difficult to learn canonization rules, which were injected by the single canonical sequence. For the $\mathrm{x} 10 \mathrm{M}$ training set, the transformer calculated $52.8 \%$, which was similar to $53.1 \%$ calculated using the $\mathrm{x} 20 \mathrm{~S}$ training dataset.

Top-5 performance analysis. This measure provided a relaxed estimation of the performance of the model by measuring if the correct reaction is listed in the top-5 predicted reactions. Actually, it is questionable whether for retrosynthetic models having the highest top-1 accuracy is desirable. The goal of a retrosynthetic model is to obtain several precursor suggestions and not exclusively the ones stated in the literature. Moreover, multiple reactions for the same product exist. An example includes the aromatic substitution of an aryl halide (R-X) to an aryl amine (R$\mathrm{NH} 2$ ) or aryl hydroxide (R-OH). Models with higher top-n scores do suggest other probable reactions (indeed, all reactions amid top-n have similar probability) which may correspond to those not reported in the literature for the analyzed example. Thus models with higher top- $\mathrm{N}$ scores but with similar top-1 scores could be interesting for a chemist since they do propose the correct prediction along with similar quality top- 1 reactions.

For each number of augmentations, the top- 5 performance generally increased with the number of augmented sequences. The highest top-5 value was consistently calculated across different scenarios for training sets with 4-5 augmentations only (Fig. 3). The highest accuracy, $78.9 \%$, was calculated for the mixture dataset using the $\mathrm{x} 5 \mathrm{M}$ training set augmentation. This number had approximately $1 \%$ higher accuracy than that calculated using the $\mathrm{x} 5 \mathrm{~S}$ training set (Fig. 3).

Reference USPTO-50 k model. For all studies we used a fixed number of epochs $N=100$. However, we needed to confirm that this was a sufficient number of epochs and to determine if we could calculate better results by training for longer. We selected the model developed with the $\mathrm{x} 5 \mathrm{M}$ training set, which provided the highest performance for top- 5 accuracy, and trained it for an additional 400 iterations (in total 500). This additional training improved top-1 accuracy to $53.3 \%$ while top-5 performance increased to $79.4 \%$ (Table 1 ) when using beam $=5$, e.g., the same as in previous analyses.

Further improvement was achieved by using a large number of augmentations, and $\mathrm{x} 100$ as the test set. With this setting the model achieved an accuracy of $53.6 \%$ and $80.8 \%$ for top- 1 and top- 5 predictions, respectively.

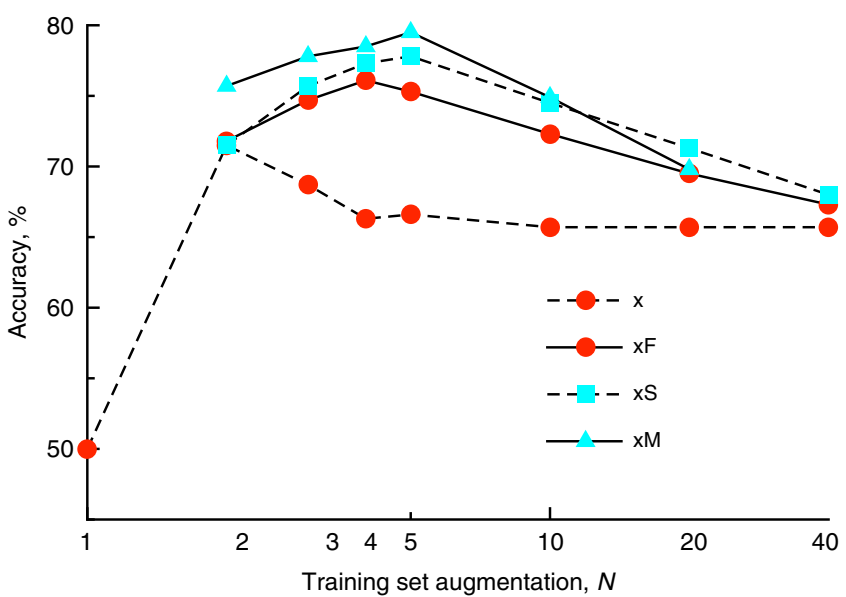

Fig. 3 Top-5 performance of transformer models developed with different training set augmentation protocols for prediction of the $\times 20$ test set. Supplementary Tables 1 and 2 for description of the respective protocols.

Influence of temperature. In our previous study ${ }^{13}$, we observed that using higher temperatures during beam search increased model accuracy for the top-1 prediction. It should be mentioned that no augmentation was used in that study. Under the same experimental setup with no augmentation, i.e., when predicting test set composed of only canonical sequences, $\mathrm{x} 1$, the top-1 accuracy of the model increased from $48.3 \%$ to $49.1 \%$ and $49.2 \%$ when using temperatures 1.3 or 1.5 , respectively. However, the top- 1 and top-5 performances for the augmented data (x20) decreased from $53.3 \%$ to $52.7 \%$ and $52.4 \%$, respectively. For the same test set the top-5 accuracies also decreased from $79.4 \%$ to $77.7 \%$ and $77.4 \%$ for both temperatures, respectively. Thus, while higher temperatures increased the variability of predictions and thus performance for prediction of canonical sequences, its effect was negative for the augmented data. In particular, it resulted in the lower accuracy of top-5 predictions.

Influence of beam search. In the above studies we consistently used a beam size of 5 for all analyses. The goal of the beam search was to generate multiple predictions for the same data and thus to better explore the variability of predictions. For example, when using the $\mathrm{x} 20$ test set and a beam size of 5 , we obtained up to 100 individual predictions, which were used to select the most frequently appearing top- 1 and top- 5 sequences. Increasing the beam size to 10 further improved top- 1 by 0.2 to $53.5 \%$ and top- 5 by $0.6 \%$ to $80 \%$ for the test set. The decrease of the beam size to 3 provided a slightly higher top- 1 score of $53.4 \%$ but decreased the top- 5 to $78.5 \%$ for the same test set. The use of beam size 1 resulted in a top-1 accuracy of $53.3 \%$ and a reduced top-5 accuracy of $75.3 \%$ (Table 1 ). These results were expected: the variation of the beam size slightly influenced the identification of the highest ranked sequence but its smaller number reduced exploration of the space of other top-reactions for larger $n$.

Both beam search and augmentation increased the number of predicted SMILES which in turn led to better accuracy of model predictions. Thus both of these methods could contribute to the generation of multiple predictions to be used to identify topranked sequences. The maximum size of the beam was restricted by the size of the target vocabulary (number of characters in the target SMILES), which was 44 characters for our dataset. Because of the design of the beam search and because we explicitly excluded duplicated predictions (see section "Analysis of predicted SMILES” as well as Supplementary Table 3), the dataset used for 
Table 1 Analysis of the reference model performance depending on the parameters of the application protocol.

\begin{tabular}{|c|c|c|c|c|c|c|c|}
\hline \multirow[b]{2}{*}{ Apply model setting } & \multicolumn{2}{|c|}{ Test set $\mathbf{x} 1$} & \multicolumn{2}{|c|}{ Test set $\mathbf{x} 20$} & \multicolumn{3}{|c|}{ Test set $\times 100$} \\
\hline & Top-1 & Top-5 & Top-1 & Top-5 & Top-1 & Top-5 & Top-10 \\
\hline Reference accuracya & 48.5 & 72.5 & 53.3 & 79.4 & 53.6 & 80.8 & 85 \\
\hline Temperature, $t=1.3$ & 49.1 & 67.7 & 52.7 & 77.7 & 53.3 & 78.4 & 83.2 \\
\hline No beam search, i.e., beam $=1$ & 47.7 & 47.7 & 53.3 & 75.3 & $53.8^{\star}$ & 78.8 & 81.7 \\
\hline Beam size, beam $=10$ & 48.3 & 73.4 & 53.5 & 80 & 53.5 & $81^{\star}$ & 85.7 \\
\hline Beam size, beam $=44$ & 48.3 & 72.5 & 53.5 & 80 & 53.5 & 80.5 & $85.8^{\star}$ \\
\hline
\end{tabular}

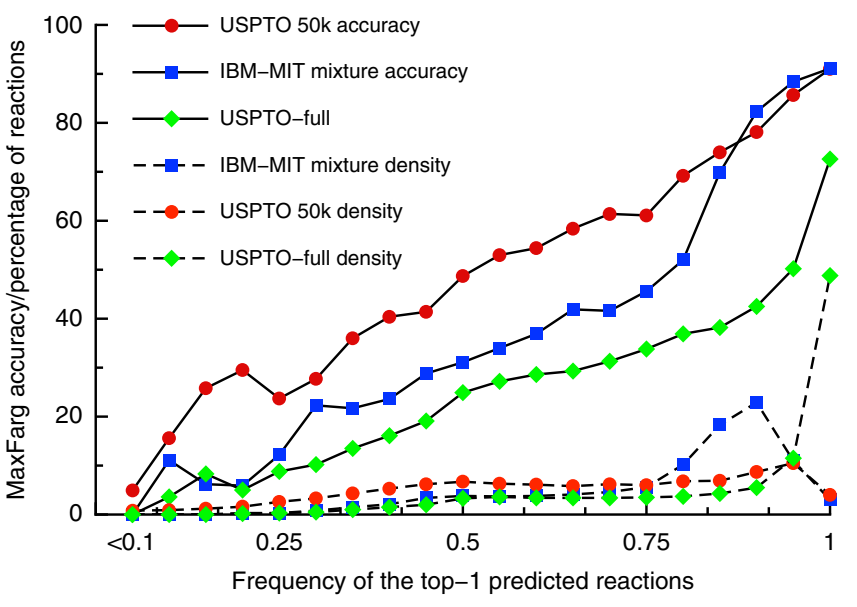

Fig. 4 Accuracy and density (fraction of predictions) of the Transformer for MaxFrag top-1 retrosynthesis accuracy. The accuracy and density are shown as a function of the frequency of appearance of the top-1 SMILES in the output of the Transformer for the respective test sets of the models.

analysis did not generate duplicated sequences for the same beam search. However, such sequences were indeed generated at different positions of the beam as different representations of the same SMILES. The number of non-unique sequences generated within the same beam search increased with the length of the beam. Interestingly, the use of canonical SMILES as input data contributed to the largest number of unique SMILES, which were $86 \%, 82 \%$ and $78 \%$ for beam searches of size 5,10 , and 44 , respectively. The use of augmented random SMILES as input contributed smaller numbers of unique sequences, e.g., $42 \%, 28 \%$ and $13 \%$ for beam searches of size 5,10 , and 44 , respectively. For both types of SMILES some generated SMILES were erroneous and could not be correctly converted by RDKit. Such sequences were excluded from analysis. For large beam sizes, canonical SMILES produced a much bigger percentage of incorrect SMILES, as compared to the use of random SMILES (see Supplementary Fig. 4). The large difference in the results generated when starting from canonical and random SMILES was also observed for analysis of the percentage of correct predictions for each beam position. In general, the number of erroneous SMILES was low, e.g., on average it was less than $1 \%$ and $3 \%$ for beam search 10 , when using augmented and canonical SMILES as input, respectively (Supplementary Fig. 4). While graph-based methods predict exact chemical structures and thus have $0 \%$ syntactically invalid SMILES, a few percentage points of incorrectly predicted structures by the transformer model does not make a large difference to these methods.
The use of canonical SMILES provided (Supplementary Fig. 4) a higher accuracy for the first beam position, but its accuracy was much lower for other beams. This was because the Transformer generated canonical SMILES for the canonical input sequences (e.g., $91 \%$ of valid SMILES produced at the position 1 of the beam search for input canonical SMILES were canonical ones) and since only one valid canonical SMILES could be produced, it failed to generate new correct SMILES. Indeed, during the training phase, the transformer always had a pair of canonical SMILES as input and target sequences. Contrary to that, using augmented SMILES allowed more freedom and allowed it to contribute valid but not necessarily canonical SMILES (e.g., only 33\% of generated SMILES at the position one of the beam search were canonical ones if augmented SMILES were used as input).

The decrease in performance of SMILES generated when using canonical SMILES was one of the main reasons to implement deduplication of data and retain only the first SMILES for the prediction of reactions (see section "Analysis of predicted SMILES"). When deduplication was not performed and all SMILES generated during the beam search were used to rank predictions (compare Supplementary Tables 3 and 4), the top-1 performances of models were most significantly affected when using only few augmentations, e.g., for the reference model its accuracy dropped from $48.3 \%$ (reference prediction, Table 1) to $47 \%$ but did not change for, e.g., top-5 performance. In principle, the analysis retaining multiple predicted sequences was based on more data and thus was more stable. Therefore, it could be used when several augmentations and/or large values of top- $n$ are used for analysis.

As it was mentioned above, both data augmentation and beam search could be used to generate multiple predictions. For the same number of generated sequences, 1000 per SMILES, using a beam $=10$ search for the x100 set produced lower accuracy, $53.5 \%$ compared to $53.7 \%$ using augmented data with the $\mathrm{x} 1000$ test set without any beam search. The performance of both methods were the same and equal to $53.7 \%$ when the deduplication procedure was not used. However, the beam search contributed to better accuracy, i.e., $81 \%$ vs. $80 \%$ and $85.7 \%$ vs. $84.3 \%$ compared to the use of augmententation alone for top- 5 and top-10, respectively. Thus, using beam search allowed a better exploration of data when suggesting several alternative reactions. In any case the augmentation was a very important part of the beam search and for the best performance, both of these approaches should be used simultaneously. We also do not exclude that optimization of the augmentation may improve its results in the future. Moreover, data augmentation used alone without a beam search contributed superior models to the beam search used without any data augmentation. 
Table 2 Prediction accuracy of the reference model for different subsets of the test set of USPTO-50k using a beam search of size 10.

Top-1

Top-5

Top-10

\begin{tabular}{|c|c|c|c|c|c|c|c|c|c|}
\hline \multirow[b]{2}{*}{$\begin{array}{l}\text { Test set } \\
\text { augmentation }\end{array}$} & & & & & \\
\hline & All & Stereo $(20 \%)$ & No stereo $(80 \%)$ & All & Stereo $(20 \%)$ & No stereo $(80 \%)$ & All & Stereo $(20 \%)$ & No stereo $(80 \%)$ \\
\hline$x 1$ & 48.3 & 44.7 & 49.2 & 73.4 & 67.3 & 74.9 & 77.4 & 71 & 79 \\
\hline$x 20$ & 53.4 & 47.3 & 55 & 80 & 73.3 & 81.9 & 84.2 & 79.2 & 85.4 \\
\hline MaxFrag, ${ }^{a} \times 1$ & 53.5 & 48.7 & 54.7 & 79.2 & 72.7 & 80.9 & 81.6 & 75.1 & 83.3 \\
\hline MaxFrag, x20 & 58.5 & 52 & 60.1 & 84.7 & 79 & 86.1 & 88.6 & 83.6 & 89.8 \\
\hline MaxFrag, x100 & $58.5^{\star}$ & 51.2 & 60.3 & $85.4^{\star}$ & 79.4 & 86.9 & $90^{\star}$ & 85.1 & 91.2 \\
\hline
\end{tabular}

aThe classical retro-synthesis accuracy was estimated as the percentage of correctly predicted largest fragments, i.e., "maximum fragment" (MaxFrag) accuracy.

* Indicate the best results.

Accuracy of prediction. For some reaction predictions without the use of augmented sequences or position at the beam search the majority of predicted sequences were identical, while for other reactions the Transformer generated as many different SMILES as possible reactants (see Supplementary Table 5). While the beam generation procedure guaranteed that each prediction had exactly the same sequence of characters, in many cases the Transformer produced multiple noncanonical instances of the same SMILES. The frequency of the appearance of the most frequent (after conversion to the canonical representation) SMILES could, therefore, indicate the confidence of the transformer in the prediction. Figure 4 indicates such frequency (which was calculated on 100x augmented dataset) correlated well with the accuracy of prediction and could be used as a confidence score for the chemist. Indeed, the reactions in which the most frequent SMILES dominated amid all predictions for Top-1 were likely to be predicted correctly. If the most frequent SMILES had low frequencies, such predictions were likely to be incorrect ones. For about $20 \%$ of the most frequent predictions, the accuracy of the retrosynthesis prediction was above $80 \%$ and for $4 \%$ more than $90 \%$. It should be mentioned, that for a practical implementation which critically depends on the speed, e.g., multistep synthesis, there is no reason to always run all 100 predictions to get the confidence estimations. One can always estimate the probability of the most frequent SMILES and its confidence interval based on a much smaller number of predictions thus decreasing the number of calculations.

As shown in Fig. 4, the same correlations were observed for two other datasets USPTO-MIT and USPTO-Full, which are analyzed in the following section. The same approach can be used for top- $n$ predictions by suggesting one or more plausible pathways for retrosynthesis. An example of such correlations for Top-5 MaxFrag accuracy were shown in Supplementary Fig. 5. Moreover, the same approach also predicted the accuracy for the direct synthesis as it was demonstrated at Supplementary Fig. 6. It should be mentioned that use of data augmentation is not the only approach to estimate the accuracy of predictions, and other methods based on the likelihood of the direct reaction prediction were also proposed ${ }^{18,34}$ and were shown to correlate with the accuracy of the predictions. A comparison of such methods is beyond the scope of this study.

Analysis of prediction accuracy for different scenarios. The accuracy of the reference model was about $5 \%$ to $7 \%$ (top-1) and $10 \%$ (top-5) higher for reactions without stereochemistry than for those with it (Table 2). $20 \%$ of the reactions in the test set contained molecules with stereochemistry. An increase in the number of augmentations of the test set increased the accuracy of both stereo and non-stereochemical reactions. Stereochemical reactions in the dataset may also suffer from a larger number of annotation errors or/and can have lower prediction scores since such data were underrepresented in the training set. In addition, for some reactions despite the relative stereochemistry being conserved it may still define confusing information for the model due to the reactant satellite effect. The R/S could be also affected by the way the SMILES was written, e.g., from $\mathrm{A}$ to $\mathrm{Z}$ or $\mathrm{Z}$ to $\mathrm{A}$.

Classical retro-synthesis accuracy: recognition accuracy for the largest fragment. The prediction of SMILES for retro-synthesis includes exact prediction of the reactants. However, the same reaction performed using different reactants can result in a similar yield. In general the database does not contain all possible reaction conditions to make a given product. Therefore, a prediction of only the main (the largest) reactant can be considered more relevant for retro-synthesis predictions, since we need to first identify the reaction type. Indeed, a chemist generally writes a retrosynthesis by decomposing a target molecule into pieces. This classical procedure, focusing only on main compound transformations, is the minimal information required to get an efficient retrosynthesis route and at the same time all reactions needed (see Fig. 5). The selection of reaction conditions of the reactions can be considered as a subsequent task.

That is why we decided to consider the recognition of the largest reactant as a new measure of the model performance: classical retro-synthesis accuracy, i.e., the accuracy of prediction of the "Maximal Fragment" (MaxFrag). The MaxFrag was $85.4 \%$ for the top-5 reaction prediction (Table 2). The MaxFrag is important to estimate an ability of a system to automatically deduce the correct reaction class. This strategy is orthogonal to explicitly providing reaction class information as input to a model ${ }^{24}$. Adding the reaction class as prior information is equivalent to getting a hint on an exam: this is impractical and also reduces the chance of proposing alternate feasible reactions. Using MaxFrag is more accurate and logical than providing a reaction class as prior information. Besides MaxFrag and Top- $n$, other scores were proposed to evaluate the success of retro suggestions/reactions, e.g., the matching score by Satoh and Funatsu ${ }^{37}$, the "in-scope" filter by Segler et al. ${ }^{5}$, and the forward transformer score by Schwaller et al. ${ }^{34}$. However, MaxFrag is the easiest and the interpretable one.

Retrosynthesis data quality and MaxFrag accuracy. The use of the classical retro-synthesis accuracy (MaxFrag top- $n$ ) calculated a systematic higher accuracy in comparison to the traditional top- $n$ scores. To explain this fact, we analyzed our datasets and found four types of reactions: non-reagent reactions, one reagent reactions, multiple reagent reactions, and unclear reagent reactions. Nonreagent reactions were reactions that $\operatorname{did}$ not work (i.e., $\mathrm{A}+\mathrm{B}->\mathrm{A}$ ). 


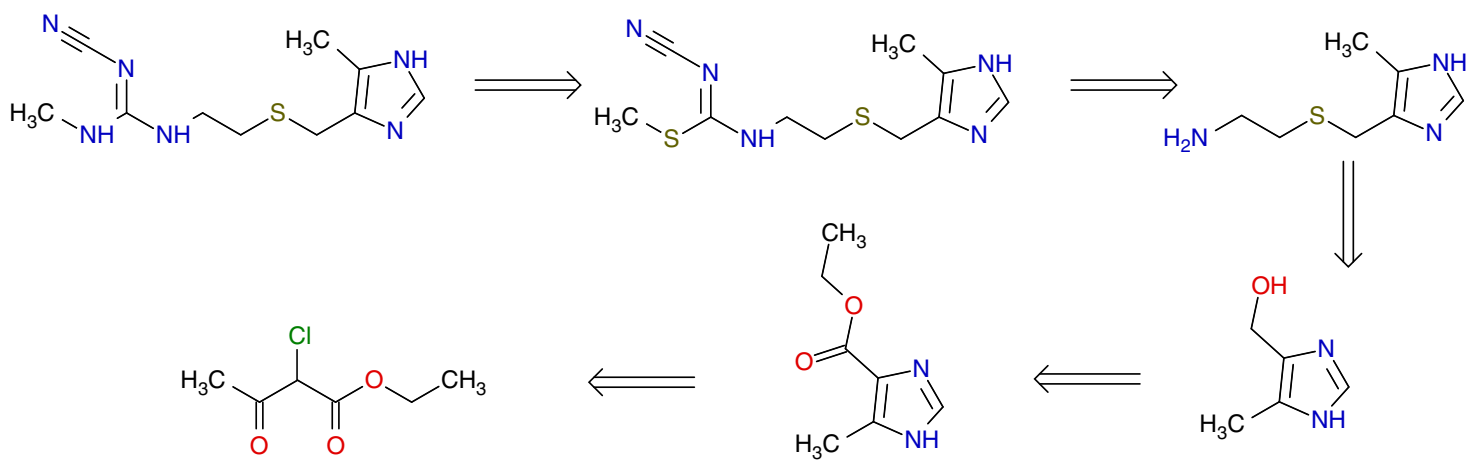

Fig. 5 Classical representation of the retrosynthesis of cimetidine focusing on the principal transformations, as is typically written by synthetic chemists (adapted from https://de.wikipedia.org/wiki/Cimetidin under CC BY-SA $\mathbf{3 . 0}$ license). The currently used top-n accuracy measures also include prediction of other reactants $12,13,19,20.24,32$, which may not be necessary for classical retrosynthesis planning.

One reagent reactions had only one starting material for the product $(\mathrm{A}->\mathrm{P})$, multiple reagents had multiple starting materials for the same end product $(\mathrm{A}+\mathrm{B}->\mathrm{P})$, and finally unclear reagents where the reaction conditions, solvent, salts, and so on, were included as reagents $(\mathrm{A}+\mathrm{B}+\mathrm{N}->\mathrm{P}$, where $\mathrm{N}$ were chemicals that did not participate to form the product). Depending on the dataset the proportions of these reaction categories slightly varied. In the MIT dataset around $0.5 \%$ of reactions were non-reagent reactions, and around $10 \%$ of the reactions were unclear reagent reactions while there were less than $1 \%$ of such reactions in the USPTO-50 k dataset. Thus, for the MIT set it would be impossible to fully predict about $10 \%$ of reactions for the retrosynthesis, since they contained chemicals " $\mathrm{N}$ " that did not form the reaction, but only conditions, solvent, etc. This more challenging problem of predicting not only the reactants but also the reagents, while still keeping diverse precursor suggestions was addressed elsewhere ${ }^{34}$. For the direct synthesis that was not a severe problem since the transformer could correctly identify and pick-up the interacting components ("A" and " $\mathrm{B}$ ") and predict the product. However, the use of Top- $n$ for retrosynthesis is questionable due to the aforementioned problem. The use of MaxFrag accuracy decreased those effects by focusing on the main reagent. That is why, in our opinion, the MaxFrag score better reflected the chemistry than Top-1 accuracy.

Still there is an unsolved challenge with this score due to the possibilities to synthesize the same products starting from multiple reagents. Both Top- $n$ and MaxFrag Top- $n$ scores were calculated by using the exact match of the predicted and target molecules. But, for example, in the reaction $\mathrm{R}-\mathrm{R} 1+\mathrm{NH} 3->\mathrm{R}-\mathrm{NH} 2$ multiples choices of $\mathrm{R} 1$, i.e., $-\mathrm{OH},-\mathrm{Cl},-\mathrm{Br},-\mathrm{I}$, or $-\mathrm{F}$, would be all correct predictions. The only difference would be the reaction rates and yields, which are not part of the prediction algorithms. Unfortunately the currently used scores, including MaxFrag, cannot yet correctly account for this problem. The problem to some extent could be alleviated by using Top/MaxFrag- $n$ instead of Top/MaxFrag1 scores: by considering multiple reagents generated by the model, we could also get the one provided in the initial reaction. Thus, the retrosynthesis task is not about getting high Top-1 accuracy. Any classical organic synthesis book, such as the famous Larock's "Comprehensive Organic Transformations" 38 indicates multiple ways to synthesize chemical compounds and this has to be reflected in the score. The classical retro-synthesis accuracy measured by MaxFrag is a first attempt to better handle those data ambiguities during the validation process and we highly encourage other users to use it. However, in order to enable a comparison with the previous studies we also reported traditional Top- $n$ scores.

Analysis of prediction accuracy for different classes. The original dataset USPTO-50 k set ${ }^{12}$ provides a reaction type label for every reaction. In total, ten reaction classes ranging from protection/deprotection, to carbon-carbon bond and heterocycle formation present the most common reactions in organic synthesis. The comparison of accuracy for each class of reactions was presented in Fig. 6. Our best model showed excellent results, outperforming the state-of-the-art Self-Corrected Transformer ${ }^{19}$. Functional group interconversion and addition, as well as carbon-carbon bond formation were the most difficult for the models to predict. It was not surprising, due to the diverse possibilities for choosing reactions and corresponding reactants for $\mathrm{C}-\mathrm{C}$ bond creation compared to more straightforward oxidation or protection where the set of groups and reactants is more narrow.

Prediction of direct reactions. The same strategy described in this work was applied to predicting direct reactions from the USPTO-MIT dataset ${ }^{22}$. We used $439 \mathrm{k}$ reactions (training and validation set were joined together) as the training set and predicted $40 \mathrm{k}$ reactions from the test set by training the transformer with the same architecture and parameters. The separated and mixed sets were used. In the separated set reactants and reagents were separated with the ">" sign while in mixed set all ">" are substituted with "." and the order of reactants and reagents was additionally shuffled. The mixed set was more difficult for training since the transformer had to identify the reaction center from a larger number of molecules. However, such a set better reflected a practical application since separation of data on reactants and reagents in some cases would not be possible without a knowledge of the target product, and thus it did provide a hint to the transformer about the reaction center. We have removed 316 reactions from the training set where the largest products had length smaller than five characters (no reactions were removed from the test set). The Transformer was training using the $\mathrm{x} 5 \mathrm{~N}$ augmentation protocol for the separated set as well as the $\mathrm{x} 5 \mathrm{~S}$ and $\mathrm{x} 5 \mathrm{M}$ protocols for the mixed set. Since it would be impractical to predict all reagents and reactants for the retrosynthesis task, which was used to additionally augment data in the $\mathrm{x} 5 \mathrm{M}$ protocol, only the largest reactant was retained as a target for the reverse reactions. Augmented test sets were predicted using beam size 10 (Table 3). For the mixed test set the order of reactants and reagents was shuffled.

As in previous studies, separation of reagent and reactants with ">" symbols contributed to a model $(\mathrm{x} 5 \mathrm{~N})$ with higher prediction scores than for models with mixed sets ( $x 5 S$ and $x 5 M)$. The additional augmentation of data using retrosynthesis reactions (x5M) did not improve the model. This could be due to the fact that the data for direct reactions were much larger and already contained sufficient information to develop accurate models. 


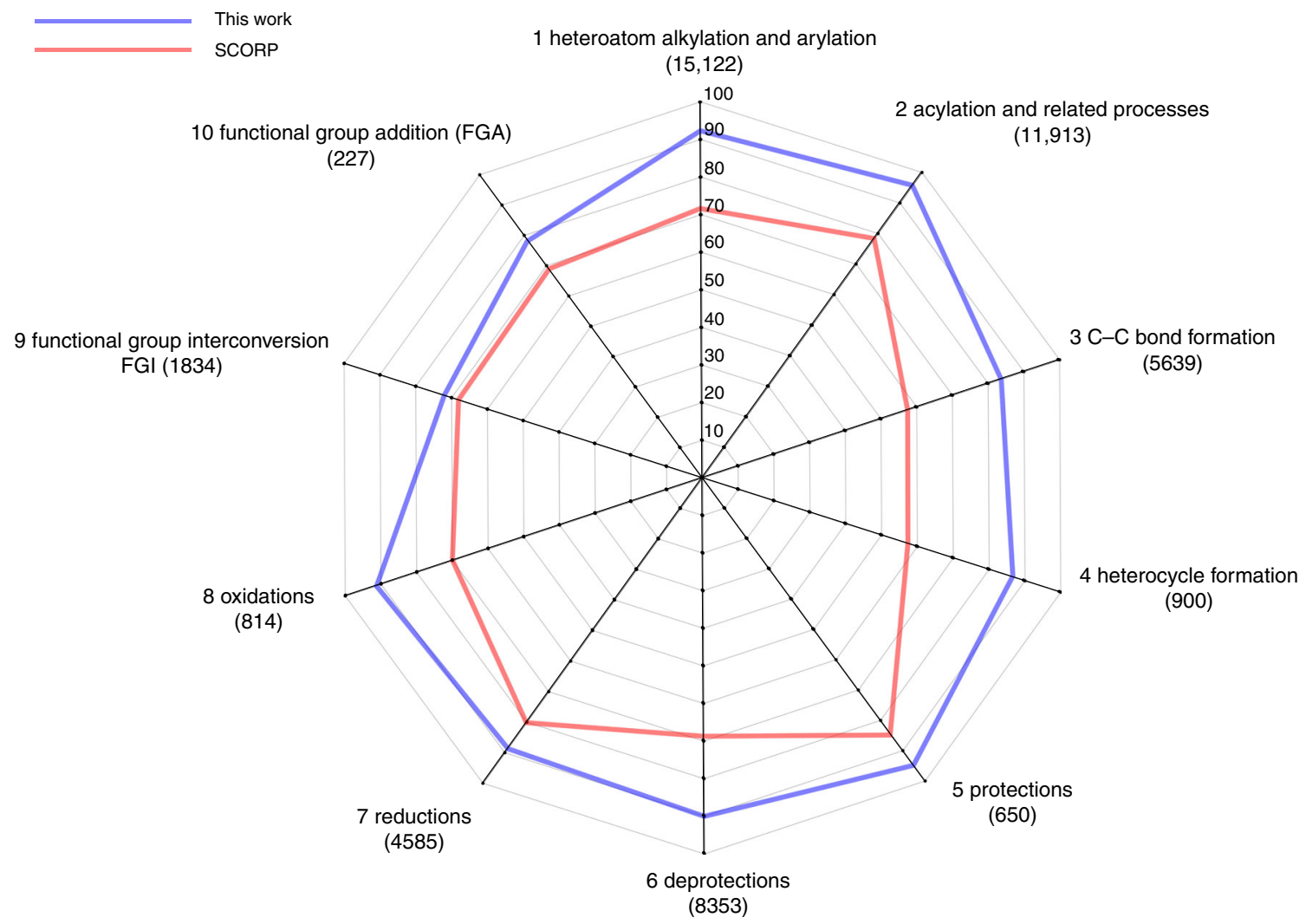

Fig. 6 Top-10 accuracy of prediction of different classes of reactions.

Table 3 Prediction accuracy for direct reaction from USPTO-MIT test set using beam size $=10$.

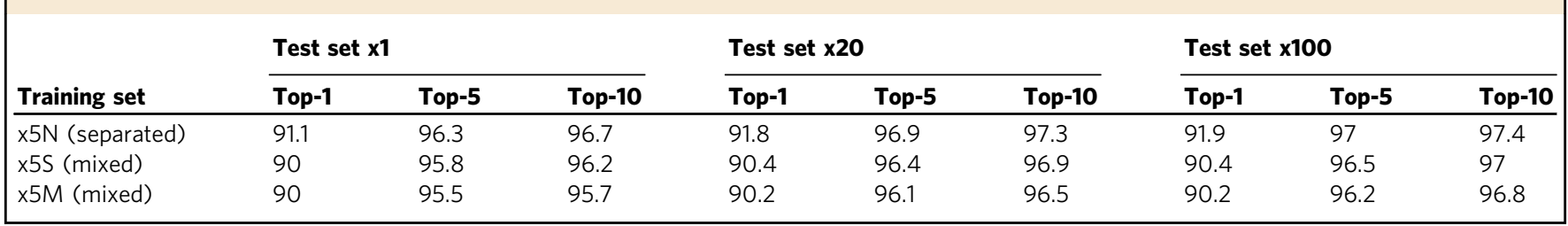

While using the x100 test set still contributed better prediction accuracy than using $\mathrm{x} 20$, the improvements were in the order of $0.1 \%$ or no improvement at all. Thus the effect of using larger augmentations on model performance reached saturation for the x100 test set.

Comparison with published models for direct synthesis using USPTO-MIT set. The USPTO-MIT was used as benchmarking for direct synthesis predictions in multiple articles. The AT provided the highest gain in performance for prediction of the more challenging mixed dataset (Table 4). Since the model was trained with randomly shuffled augmented data, it was able to generalize very well and provide excellent predictions for the new mixed data. In order to provide a more adequate comparison with previous studies we also developed a model based on exactly the same training data of $400 \mathrm{k}$. Interestingly, the use of a smaller dataset slightly increased Top-1 performance to $90.6 \%$ but decreased Top-5 performance to 96.1 . It should be noted that improvements for direct synthesis look small, i.e., just few percentages. Indeed, the model performance for the direct synthesis increased from 88.6 to 90.6 (Top-1) and 96.1 from 94.2 (Top-5) as compared to the single model reported in ref. ${ }^{18}$. Actually, this is a significant increase in performance since AT decreased the relative errors by $15 \%$ and $30 \%$ for both sets, respectively, if we consider that we can predict direct synthesis with $100 \%$. In reality we approach the experimental accuracy and further decrease of the errors will be challenging.

Comparison with published models for retrosynthesis tasks USPTO-50 $k$. The proposed augmentation protocol achieved the best published results on the USPTO-50 k dataset (Table 5). In the previous studies with this set the authors separated data on training, validation and test sets. In all our analyses, since the validation set was not used for model selection and we did not observe the model overfitting ${ }^{35}$, we joined training and validation sets to use all data in order to develop better models. While we think this is a fair comparison (it is up to the developers of the model to decide on how to best use the available data), we also added results when the model was developed with only the $40 \mathrm{k}$ compounds for USPTO-50 k set (Table 5). The accuracies of the models developed with 40 and $45 \mathrm{k}$ sets were very similar for the test set. Thus, the data augmentation allowed to compensate for the smaller size of the training set.

USPTO-MIT. We also analyzed the performance of the model at retrosynthesis of the USPTO-MIT set. Compared to USPTO-50 k this database also contained multiple reagents and possible catalysts. In our previous analysis (Table 3) we used the retrosynthesis 
Table 4 Comparison of recently published methods for direct synthesis prediction on the USPTO-MIT set.

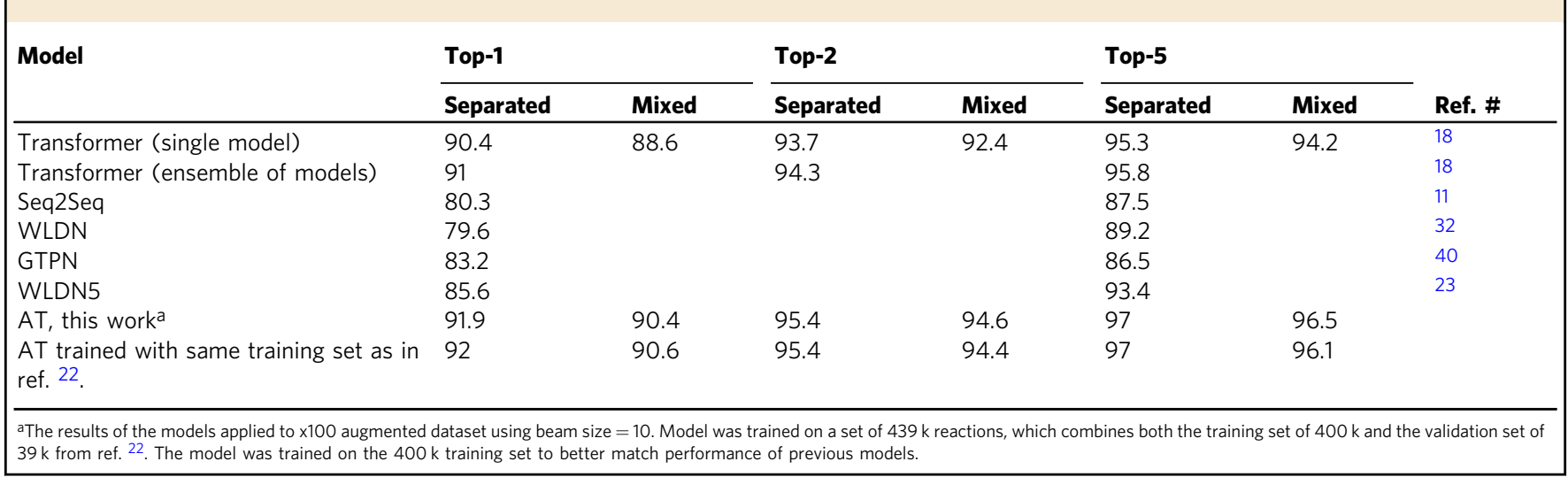

Table 5 Comparison of retrosynthesis recently published methods for retrosynthesis prediction on USPTO-50 k.

\begin{tabular}{|c|c|c|c|c|c|c|}
\hline $\begin{array}{l}\text { Transformer }\left(6^{\star} 8\right) \text {, } \\
\text { (self corrected) }\end{array}$ & 43.7 & & 65.2 & 68.7 & 19 & $40 / 5 / 5$ split, reagents from reactants are removed \\
\hline Transformer, augmentation & 44.8 & 57.1 & 57.7 & 79.4 & 32 & Same as in ref. ${ }^{12}$. \\
\hline Similarity-based & 37.3 & & 63.3 & 74.1 & 20 & Same as in ref. ${ }^{12}$. \\
\hline $\mathrm{AT}^{\mathrm{a}}$ & 53.5 & 69.4 & 81 & 85.7 & & Same as in ref. ${ }^{13}$. \\
\hline AT & 53.2 & 68.1 & 80.5 & 85.2 & & Only $40 \mathrm{k}$ samples were used as training set to match the other results \\
\hline AT MaxFragb & 58.5 & 73 & 85.4 & 90 & & Same as in ref. ${ }^{13}$. \\
\hline AT MaxFrag & 58 & 73.4 & 84.8 & 89.1 & & Only $40 \mathrm{k}$ samples were used as training set to match the other results \\
\hline
\end{tabular}

of the largest fragment as part of the "mix" protocol (x5M), i.e., the products were used as input data contained with "." to predict the largest reactant (as explained in the Supplementary materials, in order to distinguish both direct and retrosynthesis reactions, one of them started with a dot). The dot in front of the SMILES allowed the Transformer to distinguish retrosynthesis from the primary studied direct synthesis reaction. But, of course, the model trained with such data could be also used for retrosynthesis, provided that input data also started with "... We also developed a new retrosynthesis model for this set by making it more compatible to USPTO-50 k. For this we kept only the 1-2 largest fragments as the targets for retrosynthesis prediction and trained a new model using the $\mathrm{x} 5 \mathrm{~S}$ protocol. Both models were used to predict the $40 \mathrm{k}$ test set which was augmented 100 times. The MaxFrag performances of x5S model, 61.9\% (Top-1), 84.4\% (Top5 ), and $86.9 \%$ (Top-10) were very similar to those calculated for the USPTO-50 k set $(58.5,85.4$, and 90 -see Table 5). The $\mathrm{x} 5 \mathrm{M}$ model, which as aforementioned was a "by-product" of our direct reaction predictions, calculated MaxFrag of $61.1 \%, 84.4 \%$ and $88.2 \%$ for the MaxFrag Top1-,Top-5, and Top-10, respectively. Considering that the USPTO-MIT set contained more diverse reactions than USPTO-50 k, this result clearly demonstrated the excellent performance of the developed approach and its scalability. The augmented transformer (AT) was able to improve its performance for the Top-1 by extracting knowledge from a much larger dataset of reactions.
USPTO-full. The final testing was done using a USPTO-full set by Dai et al. ${ }^{24}$. The authors created a large dataset from the entire set of reactions from USPTO 1976-2016. For reactions with multiple products they duplicated them into multiple ones with one product each. The authors also removed duplications in reactions as well as those with wrong mapping to obtain train/ valid/test datasets with $800 / 100 / 100 \mathrm{k}$ sizes. Our analysis identified that some reactions in these sets were still invalid, e.g., contained no products or just single ions as reactants (e.g., US08163899B2,>>[OH2:11]; US06048982,CC $(=\mathrm{O})$ OCCCCC [I:22] >>[I-:22]; US07425593B2,>>[K:12]; US08114877B2,CC $[\mathrm{I}: 13]>>[\mathrm{I}-: 13])$. We eliminated such reactions as well as those where reactants had less than five atoms in total, since these were unlikely to be correct reactions. This procedure decreased sizes of the train/valid/test sets on average by $4 \%$ to $769 / 96 / 96 \mathrm{k}$. The AT trained using $\mathrm{x} 5 \mathrm{M}$ protocol using the $769 \mathrm{k}$ training set calculated the higher performance compared to results from the previous study (Table 6). Considering that after the removal of the $4 \%$ erroneous reactions the test dataset was decreased, we also included recalculated performance for it by assuming the worst case scenario: that AT and other tested methods failed for all excluded sequences. Even for this very conservative estimation the AT provided significant improvements compared to previously reported results. The MaxFrag accuracies for USPTOfull were lower compared to that of other analyzed sets due to the much higher diversity of this set. 


\section{Table 6 Top-k accuracy for retrosynthesis prediction on} USPTO-full dataset.

\begin{tabular}{|c|c|c|c|c|}
\hline & Retrosim 20 & Neuralsym ${ }^{3}$ & GLN $^{24}$ & $\mathbf{A} \mathbf{T}^{\mathbf{a}}$ \\
\hline Top-1 & 32.8 & 35.8 & 39.3 & $\begin{array}{l}46.2(44.4)^{b} \\
572(54.9)\end{array}$ \\
\hline $\begin{array}{l}\text { Top-2 } \\
\text { Top-10 }\end{array}$ & & & & $\begin{array}{l}57.2(54.9) \\
733(704)\end{array}$ \\
\hline $\begin{array}{l}\text { Top-10 } \\
\text { MaxFrag } \\
\text { top-1 }\end{array}$ & 56.1 & 60.8 & 63.7 & $\begin{array}{l}73.3(70.4) \\
54\end{array}$ \\
\hline $\begin{array}{l}\text { MaxFrag } \\
\text { top-2 }\end{array}$ & & & & 66.3 \\
\hline $\begin{array}{l}\text { MaxFrag } \\
\text { top-5 }\end{array}$ & & & & 77.3 \\
\hline $\begin{array}{l}\text { MaxFrag } \\
\text { top-10 }\end{array}$ & & & & 80.6 \\
\hline
\end{tabular}

a Model was trained using a filtered training set of $769 \mathrm{k}$ from ref. 20

${ }^{b}$ Accuracies in parentheses correspond to those recalculated for the test set by assuming that AT failed for all $4 \%$ of excluded reactions. Results for retrosim and neuralsym approaches as reported by Dai et al. ${ }^{24}$.

Thus for all analyzed data sets the AT provided an outstanding performance by consistently and significantly overperforming all previously published models for all statistical performances.

\section{Discussion}

This study showed that careful design of the training set was of paramount importance for the performance of the Transformer. Training the model to learn different representations of the same reaction by distorting the initial canonical data eliminated the effect of memorization and increased the generalization performance of models. These ideas are intensively used, e.g., for image recognition $^{39}$, and have been already successfully used in the context of several chemical problems ${ }^{27-30}$, including reaction predictions ${ }^{18,31}$, but were limited to the input data. For the first time we showed that application of augmentation to the target data significantly boosts the quality of the reaction prediction. We also showed for the first time that the frequency of predicted SMILES could be used as a confidence metric for (retro)synthesis prediction and can provide quantitative estimation of the most probable reactions amid top-n predicted outcomes. It is very critical to estimate the quality of the reaction prediction since it could help to better prioritize multi-step retrosynthesis. The developed methodology is unique to the use of augmentation techniques, currently unavailable to $\mathrm{GCNs}^{24}$, which directly operates with graphs. The estimated accuracy of prediction can help to distinguish reactions, which are difficult to predict, from typo and erroneous reaction data, which will be important to clean up the reaction data and further improve model quality. We also introduced a new MaxFrag measure, classical retro-synthesis accuracy, which in our opinion better reflects the requirements for retrosynthesis analysis.

It should be mentioned that use augmentation was first studied by authors of ref. ${ }^{18}$, who introduced transformer to chemistry and applied it to chemical reactions by using SMILES instead of the text sequences. The augmentation of input data, which was done in that article, provided only a minor improvement of their models. Because of its small impact it was not followed in several other Transformer-based works, including our own study ${ }^{13,19}$. In this article we brought an original idea on how to augment chemical data, which provided a significant improvement of the results for all analyzed datasets.

SMILES random augmentation had the ability to stabilize the model's learning by adding more data and adding more randomness and freedom into the network. Remarkably, this augmentation functioned similarly to ensemble learning, allowing for better statistics and improving the performance of the model. Beam search and augmentation were complementary and our reference model in essence got better results than models developed using graph representation of molecules ${ }^{24}$, for which the use of similar data augmentation technique is currently not possible.

\section{Data availability}

Data and predictions that support the results of this study are available at https://github. com/bigchem/synthesis.

\section{Code availability}

Source code to perform the data augmentation is available at https:/github.com/ bigchem/synthesis.

Received: 19 March 2020; Accepted: 5 October 2020; Published online: 04 November 2020

\section{References}

1. Corey, E. J. \& Cheng, X.-M. The Logic of Chemical Synthesis. (John Wiley \& Sons, New York, 1995).

2. Corey, E. J., Long, A. K. \& Rubenstein, S. D. Computer-assisted analysis in organic synthesis. Science 228, 408-418 (1985).

3. Segler, M. H. S. \& Waller, M. P. Neural-symbolic machine learning for retrosynthesis and reaction prediction. Chemistry 23, 5966-5971 (2017).

4. Coley, C. W., Barzilay, R., Jaakkola, T. S., Green, W. H. \& Jensen, K. F. Prediction of organic reaction outcomes using machine learning. ACS Cent. Sci. 3, 434-443 (2017)

5. Segler, M. H. S., Preuss, M. \& Waller, M. P. Planning chemical syntheses with deep neural networks and symbolic AI. Nature 555, 604-610 (2018).

6. Baskin, I. I., Madzhidov, T. I., Antipin, I. S. \& Varnek, A. A. Artificial intelligence in synthetic chemistry: achievements and prospects. Russian Chem. Rev. 86, 1127-1156 (2017).

7. Struble, T. J. et al. Current and future roles of artificial intelligence in medicinal chemistry synthesis. J. Med. Chem. 63, 8667-8682 (2020).

8. Muratov, E. N. et al. QSAR without borders. Chem. Soc. Rev. 49, 3525-3564 (2020).

9. Szymkuć, S. et al. Computer-assisted synthetic planning: the end of the beginning. Angew. Chem. Int. Ed. Engl. 55, 5904-5937 (2016).

10. Law, J. et al. Route Designer: a retrosynthetic analysis tool utilizing automated retrosynthetic rule generation. J. Chem. Inf. Model. 49, 593-602 (2009).

11. Schwaller, P., Gaudin, T., Lanyi, D., Bekas, C. \& Laino, T. "Found in Translation": predicting outcomes of complex organic chemistry reactions using neural sequence-to-sequence models. Chem. Sci. 9, 6091-6098 (2018).

12. Liu, B. et al. Retrosynthetic reaction prediction using neural sequence-tosequence models. ACS Cent. Sci. 3, 1103-1113 (2017).

13. Karpov, P., Godin, G. \& Tetko, I. V. In A Transformer Model for Retrosynthesis, Artificial Neural Networks and Machine Learning-ICANN 2019: Workshop and Special Sessions, Münich, 17-19th September 2019. (eds Tetko, I. V., Kůrková, V., Karpov, P. \& Theis, F.). 817-830 (Springer International Publishing, Münich, 2019).

14. Weininger, D. Smiles, a chemical language and information-system.1. Introduction to methodology and encoding rules. J. Chem. Inf. Comput. Sci. 28, 31-36 (1988).

15. Nam, J. \& Kim, J. Linking the neural machine translation and the prediction of organic chemistry reactions. Preprint at https://arxiv.org/abs/1612.09529 (2016).

16. Sutskever, I., Vinyals, O. \& Le, Q. V. In Sequence to Sequence Learning with Neural Networks, Advances in Neural Information Processing Systems vol 27. (eds Ghahramani, Z., Welling, M., Cortes, C., Lawrence, N. D. \& Weinberger, K. Q.). 3104-3112 (Curran Associates, Inc., 2014).

17. Vaswani, A. et al. Attention Is All You Need. Preprint at https://arxiv.org/abs/ 1706.03762 (2017).

18. Schwaller, P. et al. Molecular transformer: a model for uncertainty-calibrated chemical reaction prediction. ACS Cent. Sci. 5, 1572-1583 (2019).

19. Zheng, S., Rao, J., Zhang, Z., Xu, J. \& Yang, Y. Predicting retrosynthetic reactions using self-corrected transformer neural networks. J. Chem. Inf. Model. 60, 47-55 (2020).

20. Coley, C. W., Rogers, L., Green, W. H. \& Jensen, K. F. Computer-assisted retrosynthesis based on molecular similarity. ACS Cent. Sci. 3, 1237-1245 (2017).

21. Ishida, S., Terayama, K., Kojima, R., Takasu, K. \& Okuno, Y. Prediction and interpretable visualization of retrosynthetic reactions using graph convolutional networks. J. Chem. Inf. Model. 59, 5026-5033 (2019). 
22. Jin, W., Coley, C., Barzilay, R. \& Jaakkola, T. In Predicting Organic Reaction Outcomes with Weisfeiler-Lehman Network, 31st Conference on Neural Information Processing Systems (NIPS 2017), Long Beach, CA, USA. (eds Guyon, I., Luxburg, U. V., Bengio, S., Wallach, H., Fergus, R., Vishwanathan, S. \& Garnett, R.). 2607-2616 (Long Beach, CA, USA, 2017).

23. Coley, C. W. et al. A graph-convolutional neural network model for the prediction of chemical reactivity. Chem. Sci. 10, 370-377 (2019).

24. Dai, H., Li, C., Coley, C., Dai, B. \& Song, L. In Retrosynthesis Prediction with Conditional Graph Logic Network, Advances in Neural Information Processing Systems vol 32. (eds Wallach, H. et al.). 8872-8882 (Curran Associates, Inc., 2019).

25. Shi, C., Xu, M., Guo, H., Zhang, M. \& Tang, J. A graph to graphs framework for retrosynthesis prediction. Preprint at https://arxiv.org/abs/2003.12725 (2020).

26. Weininger, D., Weininger, A. \& Weininger, J. L. Smiles.2. Algorithm for generation of unique smiles notation. J. Chem. Inf. Comput. Sci. 29, 97-101 (1989).

27. Tetko, I. V., Karpov, P., Bruno, E., Kimber, T. B. \& Godin, G. In Augmentation Is What You Need!, Artificial Neural Networks and Machine Learning-ICANN 2019: Workshop and Special Sessions, Münich, 17-19th September 2019. (eds Tetko, I. V., Kůrková, V., Karpov, P. \& Theis, F.). 831-835 (Springer International Publishing, Münich, 2019).

28. Kimber, T. B., Engelke, S., Tetko, I. V., Bruno, E. \& Godin, G. Synergy Effect between Convolutional Neural Networks and the multiplicity of SMILES for improvement of molecular prediction. Preprint at https://arxiv.org/abs/ 1812.04439 (2018).

29. Bjerrum, J. E. SMILES enumeration as data augmentation for neural network modeling of molecules. Preprint https://arxiv.org/abs/1703.07076 (2017).

30. Karpov, P., Godin, G. \& Tetko, I. V. Transformer-CNN: Swiss knife for QSAR modeling and interpretation. J. Cheminform. 12, 17 (2020).

31. Fortunato, M. E., Coley, C. W., Barnes, B. C. \& Jensen, K. F. Data augmentation and pretraining for template-based retrosynthetic prediction in computer-aided synthesis planning. J. Chem. Inf. Model. 60, 3398-3407 (2020).

32. Chen, B., Shen, T., Jaakkola, T. S. \& Barzilay, R. Learning to make generalizable and diverse predictions for retrosynthesis. Preprint at https:// arxiv.org/abs/1910.09688 (2019).

33. Lin, K., Xu, Y., Pei, J. \& Lai, L. Automatic retrosynthetic route planning using template-free models. Chem. Sci. 11, 3355-3364 (2020).

34. Schwaller, P. et al. Predicting retrosynthetic pathways using transformer-based models and a hyper-graph exploration strategy. Chem. Sci. 11, 3316-3325 (2020).

35. Tetko, I. V., Livingstone, D. J. \& Luik, A. I. Neural network studies. 1. Comparison of overfitting and overtraining. J. Chem. Inf. Comput. Sci. 35, 826-833 (1995).

36. Lowe, D. M. Extraction of Chemical Structures and Reactions from the Literature (University of Cambridge, 2012).

37. Satoh, H. \& Funatsu, K. SOPHIA, a knowledge base-guided reaction prediction system - utilization of a knowledge base derived from a reaction database. J. Chem. Inf. Comput. Sci. 35, 34-44 (1995).

38. Larock, R. C Comprehensive Organic Transformations: A Guide to Functional Group Preparations (John Wiley \& Sons: Hoboken, NJ, 1999).

39. Shorten, C. \& Khoshgoftaar, T. M. A survey on image data augmentation for deep learning. J. Big Data 6, 60 (2019).

40. Do, K., Tran, T. \& Venkatesh, S. Graph transformation policy network for chemical reaction prediction. In Proceedings of the 25th ACM SIGKDD International Conference on Knowledge Discovery \& Data Mining, Association for Computing Machinery: Anchorage, AK, USA, 750-760 (2019).

\section{Acknowledgements}

This study was partially funded by the European Union's Horizon 2020 research and innovation program under the Marie Skłodowska-Curie Innovative Training Network European Industrial Doctorate grant agreement No. 676434, "Big Data in Chemistry" and ERACVD "CardioOncology" project, BMBF 01KL1710 as well as by a grant from Intel. The article reflects only the author's view and neither the European Commission nor the Research Executive Agency (REA) are responsible for any use that may be made of the information it contains. The authors thank NVIDIA Corporation for donating Quadro P6000, Titan Xp, and Titan V graphics cards for this research work. The authors thank Michael Withnall (Apheris AI) and Alli Michelle Keys (Stanford University) for their comments and English corrections as well as Marios Theodoropoulos (University of Geneva) for interesting discussions. We also would like to thank the anonymous reviewers for their insightful and sometimes even provocative comments answering of which significantly increased the value of this study.

\section{Author contributions}

G.G., R.v.D., and I.V.T. initially designed the study and proposed several different augmentation protocols and started initial analysis. I.V.T. and P.K. performed the advanced analysis. All authors contributed equally to the paper. All authors read and approved the final paper.

\section{Funding}

Open Access funding enabled and organized by Projekt DEAL.

\section{Competing interests}

The authors declare no competing interests.

\section{Additional information}

Supplementary information is available for this paper at https://doi.org/10.1038/s41467020-19266-y.

Correspondence and requests for materials should be addressed to I.V.T. or G.G.

Peer review information Nature Communications thanks Pierre Baldi and the other, anonymous, reviewers for their contribution to the peer review of this work.

Reprints and permission information is available at http://www.nature.com/reprints

Publisher's note Springer Nature remains neutral with regard to jurisdictional claims in published maps and institutional affiliations.

Open Access This article is licensed under a Creative Commons Attribution 4.0 International License, which permits use, sharing, adaptation, distribution and reproduction in any medium or format, as long as you give appropriate credit to the original author(s) and the source, provide a link to the Creative Commons license, and indicate if changes were made. The images or other third party material in this article are included in the article's Creative Commons license, unless indicated otherwise in a credit line to the material. If material is not included in the article's Creative Commons license and your intended use is not permitted by statutory regulation or exceeds the permitted use, you will need to obtain permission directly from the copyright holder. To view a copy of this license, visit http://creativecommons.org/ licenses/by/4.0/.

(C) The Author(s) 2020 